\title{
AVALWEB - Sistema interativo para gerência de questões e aplicação de avaliação na Web
}

\author{
Carlos Morais ${ }^{2}$, Jose Valdeni de Lima ${ }^{1}$, Sérgio Roberto Kieling Franco ${ }^{2 .}$ \\ ${ }^{1}$ Instituto de Informática - Universidade Federal do Rio Grande do Sul (UFRGS) \\ Caixa Postal 15.064 - 91.501-970 - Porto Alegre - RS - Brazil \\ ${ }^{2}$ Programa de Pós-Graduação de Informática na Educação (PPGIE//UFRGS), \\ \{cmorais, valdeni\} dinf.ufrgs.br, francodedu.ufrgs.br
}

\begin{abstract}
This paper presents a tool developed with apply free software for study the processes of evaluation of student in environment of Distance Education. Several Evaluation tools available in the market were analyzed to attain such a goal. Based on said analyses, a query-management and assessment application was proposed. The system allows the pupil to the self-evaluation, immediate feedback of the result, integrating the system of management of questions with explanations of reasons hits and mistakes achieved in the answering process. Giving emphasis more in the process of learning/teaching more than the assessment probably. Finally, a prototype was implemented to show how viable the ideas presented in the evaluation model herein proposed are. The conceptual proposal, which was established for the model, is far broader and more pliable than the existing version of achieved implementation.
\end{abstract}

\begin{abstract}
Resumo. Este artigo apresenta uma ferramenta desenvolvida com software livre aplicado ao estudo dos processos de avaliação de alunos em ambiente de Ensino a Distância. Para tanto, foram analisadas várias ferramentas de avaliação disponíveis no mercado. Com estas análises, foi modelado o sistema de gerência de questões e avaliações. O sistema permite ao aluno à auto-avaliação, com retorno imediato do resultado, integrando o sistema de gerência de questões com explicações do sucesso ou erro nas respostas, dando ênfase mais no processo de ensinolaprendizagem do que na avaliação propriamente dita. Enfim, foi implementado um protótipo demonstrando a viabilidade das idéias presentes no modelo de avaliação aqui apresentado. A proposta conceitual estabelecida para o modelo é bem mais ampla e flexível do que a atual versão da implementada.
\end{abstract}

\section{Introdução}

O apelo do uso da tecnologia na área de educação é tão marcante que alguns observadores arriscam afirmar que o e-learning ou ensino eletrônico é a mais nova onda da Internet, depois do comércio eletrônico. O IDC (International Data Corporation) estima que o mercado de treinamento à distância por meio digital vai crescer mundialmente cerca de $69 \%$ ao ano. Com esse fator de crescimento, o setor deve sair de um patamar de modestos 2 bilhões de dólares em 1999 para 23 bilhões de dólares em 2004. De acordo com estas previsões, a demanda atual e futura desta área justifica a criação de ferramentas que possam ser aplicadas neste contexto (Moreira 2001). Além do enorme crescimento previsto para ensino a distância, a necessidade do desenvolvimento de novas ferramentas decorre da constatação de que as idéias por trás dos sistemas ainda não estão consolidadas e o número de boas experiências no uso do computador no processo de ensino/aprendizagem é menor do que a sociedade poderia esperar. Em vista dos benefícios que podem ser obtidos, fica evidente a necessidade e oportunidade do 
desenvolvimento de técnicas e ferramentas que proporcionem a elaboração de avaliações por parte dos professores de forma que possam ser colhidos melhores resultados através de sua aplicação. O tempo e esforço necessários para produzir ferramentas de aprendizado e avaliações on-line podem ser substanciais. Esta dificuldade está presente especialmente em determinadas áreas, porque técnicas de avaliação não podem ser extraídas diretamente de livros texto e materiais de cursos (Renshaw 2000).

\section{Estado-da-arte}

Existem diversos ambientes e ferramentas específicas que abordam como complemento ou de forma única o aspecto de avaliação dos alunos. Para melhor embasamento, foram analisados produtos considerados líderes em seu segmento de mercado com fortes características avaliativas. Através dos ambientes e ferramentas apresentados, pode-se observar que existem diversos enfoques sobre a questão da avaliação, mas de todas as formas, também há determinado padrão nas avaliações, como os tipos de questões apresentadas para os alunos. Os principais sistemas existentes de avaliação computadorizada são parte de ambientes Computer Based Training (CBT) e pacotes de autoria multimídia como o Asymetrix Toolbook (Tinoco 1997). Estes sistemas normalmente vêm com interface gráfica de autoria e suporte a bancos de dados de cursos, no entanto, cada um destes sistemas possui seus próprios "padrões" (por exemplo, plataformas, linguagens de autoria, representação interna dos dados). Além disto, são muito caros para serem utilizados em larga escala, como no ambiente universitário e seus custos de desenvolvimento também são excessivamente altos.

2.1 Avaliação em ambientes completos

Para validar as idéias propostas e pesquisar os ganhos obtidos com a utilização de uma ferramenta com abordagem educacional, inicialmente foram analisados aspectos que tratam da avaliação de alunos, tanto em ambientes completos de educação à distância (Aulanet, WebCt e TopClass) como em ferramentas específicas para avaliação (Question Mark Perception, Hot Potatoes e Quiz Center) (Morais 2002) (Cardoso 2001) e uma ferramenta de autoria multimídia que possibilita a criação de questionários na Web (Asymetrix Toolbook).

\section{Ferramenta AVALWEB}

Este seção apresenta a seguir alguns conceitos e metodologia do sistema apresentado, naturalmente, não é necessário usar equipamentos de processamento de dados para organizar, selecionar e arquivar questões. Estas tarefas são rotineiras e podem ser feitas à mão (Bloom 1983). Entretanto, há um ponto - que deve ser determinado em cada situação - em que a economia de tempo e o aumento no nível de exatidão no arquivamento e na manutenção da ordem tornam o uso de computadores um instrumento auxiliar precioso a um sistema de avaliação. Seu uso elimina a dificuldade ou o desconforto que muitos professores sentem quando solicitados a realizar a análise estatística dos testes. A máquina altera o papel do professor, que passa de produtor a consumidor de dados de análise de testes e de itens (Bloom 1983). O AvalWeb é um sistema que possui como foco principal o gerenciamento de questões e aplicação de avaliações via Web com base em requisições de professores. Também possui um módulo de auto-avaliação, com retorno imediato para o aluno, que integra o sistema de gerência de questões dando ênfase mais no processo de ensino/aprendizagem do que na avaliação propriamente dita.

O ensino resultante de autênticas tarefas em um ambiente aberto não é fácil de avaliar com testes tradicionais ou instrumentos existentes, portanto uma das preocupações do AvalWeb é fornecer subsídios para ajudar o processo de avaliação através de dados estatísticos (Safoutin 2000). A avaliação inclui uma grande variedade de dados, superior ao rotineiro exame escrito final (Haydt 1997). Por este motivo, o AvalWeb também procura colher diversas informações adicionais que colaboram para este propósito como, por exemplo, o tempo gasto pelo aluno para responder uma ou um conjunto de questões. O modelo proposto é baseado em uma ferramenta e não possui nenhum tipo de característica que irá assegurar automaticamente a qualidade dos cursos que serão oferecidos através de sua utilização. Existem diversas ferramentas e serviços disponíveis para finalidades correlatas mais abrangentes e que podem ser agregados, 
complementados e adaptados ao cenário obtido como resultado deste trabalho de pesquisa (Cardoso 2001).

\subsection{Arquitetura do Sistema}

A arquitetura proposta para o AvalWeb é mostrada graficamente na figura 1.0. O acesso para alunos e professores ocorre através de requisições http para o servidor, que interage com o código $P H P$, que por sua vez faz o acesso aos dados armazenados no banco de dados Mysql (Cardoso 2001).

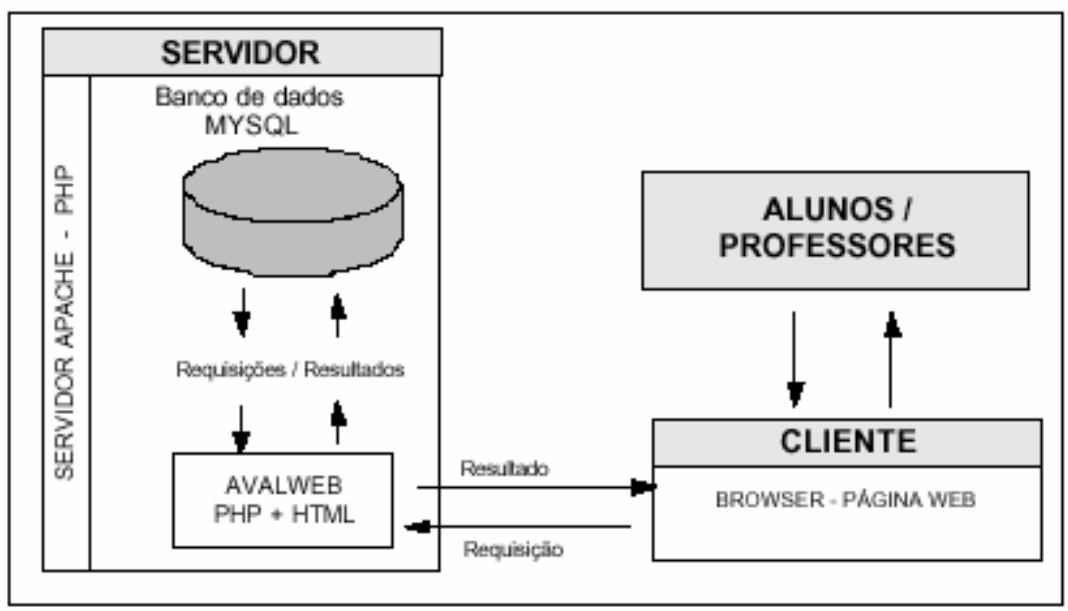

Figura 1. Arquitetura do Sistema AVALWEB

O modelo proposto está baseado na arquitetura do tipo cliente-servidor, onde o servidor Web representa a figura do banco de questões e alunos e professores são representados através de clientes utilizando seus navega dores. O código PHP está mesclado com códigos HTML, no entanto, o conteúdo em PHP não é visível ao usuário, sendo processado no servidor. (Cardoso 2001). Não é razoável esperar que os professores tenham tempo ou treino estatístico necessário à consecução de uma análise de itens e de testes. Além disso, muitos deles não estão, justificadamente, interessados em adquirir esta habilidade. Portanto, de maneira geral, os professores não submetem seus testes a uma análise técnica, apesar de um sistema formal de avaliações exigir que esta análise seja feita. $\mathrm{O}$ acesso à ferramenta via WEB e as informações relativas aos alunos, professores e demais tabelas do sistema são armazenada no servidor.

\subsection{Público envolvido}

O AvalWeb pode ser aplicado aos dois últimos cenários sugeridos anteriormente, podendo ser utilizado como ferramenta de apoio para professores do ensino tradicional ou como complemento a cursos fornecidos totalmente via WEB. (Cardoso 2001).

\section{Considerações Finais}

Apesar de já ter sido testada em alguns casos reais, a utilização da metodologia deve ser intensificada para melhor avaliação e refinamento. Para isso, é imprescindível realizar a validação com professores e também com um maior número de alunos. Com base na estrutura dos documentos e nos contextos, é definida a estrutura de navegação do sistema, especificando o relacionamento entre documentos e os caminhos de acesso às funções, permitindo o sistema interagir com outros sistemas. Estando definidas as estruturas de dados e de navegação do sistema, com isso serão analisados o comportamento dinâmico de documentos e links e como será realizada a integração entre documentos e bancos de dados. Para que todo esse processo aconteça no plano operacional, há necessidade de que, a partir de fundamentos teóricos claramente identificados e de mecanismos de exercícios e avaliações coerentes e eficazes, se promova à necessária mudança de consciência, de métodos, de instrumentos e de perspectivas. Isto implica, além de análise crítica, capacidade de formular propostas alternativas. Pretende se efetuar uma integração do AvalWeb com outros Sistemas na Web utilizando os tradicionais links de interesses dos usuários, o que torna o acesso à informação uma tarefa difícil e demorada. A 
principal causa desta situação é à falta de estruturação dos dados e a ausência de uma representação semântica dos mesmos. A Web Semântica surge para facilitar o acesso à informação através da estruturação e da representação semântica dos dados, aumentando assim a eficiência e precisão nas pesquisas efetuadas pelos usuários. Neste sentido, esta é uma proposta alternativa, que, aplicável para o ensino, e para um projeto EAD, pode ser considerada também uma visão de avanço do aprendizado, e uma alternativa para contribuir na busca de novos caminhos no contexto da pesquisa e inovação.

\section{Referências bibliográficas}

BLOOM, Benjamim S. et al. Manual de avaliação formativa e somativa do aprendizado escolar. 1 ed. São Paulo: Pioneira, 1983. 307 p.

CARDOSO, Rodrigo Ferrugem. AvalWeb - Sistema interativo para gerência de questões e aplicação de avaliações na Web 2001. Dissertação (Mestrado em Ciência da Computação) Instituto de Informática, Universidade Federal do Rio Grande do Sul, Porto Alegre.

HAYDT, Regina Cazux. Avaliação do processo Ensino-Aprendizagem. São Paulo: Ática. 1997.

MORAIS, Carlos Tadeu Q. de, Hyper-Automaton: Avaliação Interativa de Alunos em Cursos na WEB Baseado em Autômatos Finitos 2002. Dissertação (Mestrado em Ciência da Computação) - Instituto de Informática, Universidade Federal do Rio Grande do Sul, Porto Alegre.

MOREIRA, Maria Isabel. “O tema de hoje é e-learning”. Info. Exame. N. 181. Abril de 2001. p. 78.

RENSHAW, Anthony A. et al. An Assessment of On-line Engineering Design Problem Presentation Strategies. IEEE transactions on education. New York. v. 43, n. 2, p. 83-91. May 2000.

SAFOUTIN, Michael J. et al. A Design Attribute Framework for Course Planning and Learning Assessment. IEEE transactions on education. New York. v. 43, n. 2, p. 188-194. May 2000.

TINOCO, Lúcio C. et. al. On-line evaluation in WWW-based Courseware.In: Communications of ACM. p. 194-198.SIGCSE 97. 1997. 